\title{
COVID-19 Associated Miller Fisher Syndrome and Rhombencephalitis with Magnetic Resonance Imaging Findings
}

\author{
(D) Onur Akan1, (D) Begüm Yaralıoğlu11, (D) Sümeyye Karabıyık2, (D) Funda Şimşek2, (D) Şule Vatansever3, (D) Kemal Harmancı4, \\ (iD) Serap Üçler 1 \\ 1 University of Health Sciences Turkey, Prof. Dr. Cemil Taşcıoğlu City Hospital, Clinic of Neurology, Istanbul, Turkey \\ 2 University of Health Sciences Turkey, Prof. Dr. Cemil Taşcıoğlu City Hospital, Clinic of Infectious Disease, İstanbul, Turkey \\ 3 University of Health Sciences Turkey, Prof. Dr. Cemil Taşcıoğlu City Hospital, Clinic of Anesthesiology and Reanimation, Istanbul, Turkey \\ «University of Health Sciences Turkey, Prof. Dr. Cemil Taşcıoğlu City Hospital, Clinic of Radiology, Istanbul, Turkey
}

\section{Abstract}

Coronaviruses, including severe acute respiratory syndrome-coronavirus 2, are responsible for upper respiratory tract infections. However, recent studies have reported that patients with coronavirus disease-2019 (COVID-19) may manifest neurological symptoms. The involvement of the neurological system causes different clinical situations, including meningitis, encephalitis, acute necrotizing hemorrhagic encephalopathies, acute cerebrovascular events, and Guillain-Barré syndrome. Miller Fisher syndrome and rhombencephalitis were separately reported in few cases of COVID-19. In this report, we present the neuroimaging findings of a patient with Miller Fisher syndrome and rhombencephalitis due to COVID-19.

Keywords: Encephalitis, encephalopathy, rhombencephalitis, COVID-19, SARS-CoV-2, Guillian Barré syndrome, Miller Fisher syndrome, neurological complications

\section{INTRODUCTION}

Severe acute respiratory syndrome-coronavirus 2 (SARS-CoV2)-associated coronavirus disease-2019 (COVID-19) was first documented in patients with unusual pneumonia in December 2019 at Wuhan, China (1). This novel infection was promptly spread throughout the world, thus becoming a serious global health problem. In March 2020, COVID-19 was declared a pandemic by World Health Organization. Coronaviruses, including SARS-CoV-2, are responsible for upper respiratory tract infections. However, recent studies have reported that patients may manifest neurological symptoms, such as headache, dizziness, myalgia, hyposmia/anosmia, and hypogeusia/ageusia (2).

The involvement of the neurological system causes different clinical situations, including meningitis, encephalitis, acute necrotizing hemorrhagic encephalopathies, acute cerebrovascular events, and Guillain-Barré syndrome (GBS). Miller Fisher syndrome, a GBS variant, is characterized by acute ophthalmoplegia, gait ataxia, and areflexia. Rhombencephalitis refers to inflammatory disorders that affect the hindbrain (brainstem and cerebellum) (3). Miller Fisher syndrome and rhombencephalitis were separately reported in few cases of COVID-19. In this report, we present the neuroimaging findings of a patient with Miller Fisher syndrome and rhombencephalitis due to COVID-19.

\section{CASE PRESENTATION}

A 35-year-old male patient was admitted in our emergency department with complaints of fever and shortness of breath. 
He was hospitalized due to worsening respiratory symptoms and poor general condition. Nasopharyngeal swab test for COVID-19 was positive. He has an unremarkable medical history. He rapidly developed speech disturbance, verbal clumsiness, blurred vision, gait disorder, and loss of balance. Two days later, the patient suffered from swallowing difficulty. On neurologic examination, the patient was conscious and well-oriented to time, place, and person. His pupils were equal size in both eyes. The direct and indirect light reflexes were normal. He had an adduction deficit in his right eye. The abduction deficit was remarkable in both eyes without double vision. The muscle strengths were normal in all extremities. There was no sensorial deficit. All deep tendon reflexes were absent. Plantar responses were flexor. He had an ataxic gait and a positive Romberg sign. Meningeal irritation signs were absent. Cerebrospinal fluid examination showed high protein $(792.6 \mathrm{mg} / \mathrm{L})$ and normal glucose levels with leukocyte counts $\left(2 / \mathrm{mm}^{3}\right)$. Cerebrospinal polymerase chain reaction (PCR) for 2019-nCoV RNA and ganglioside GQ1b-IgG antibody was
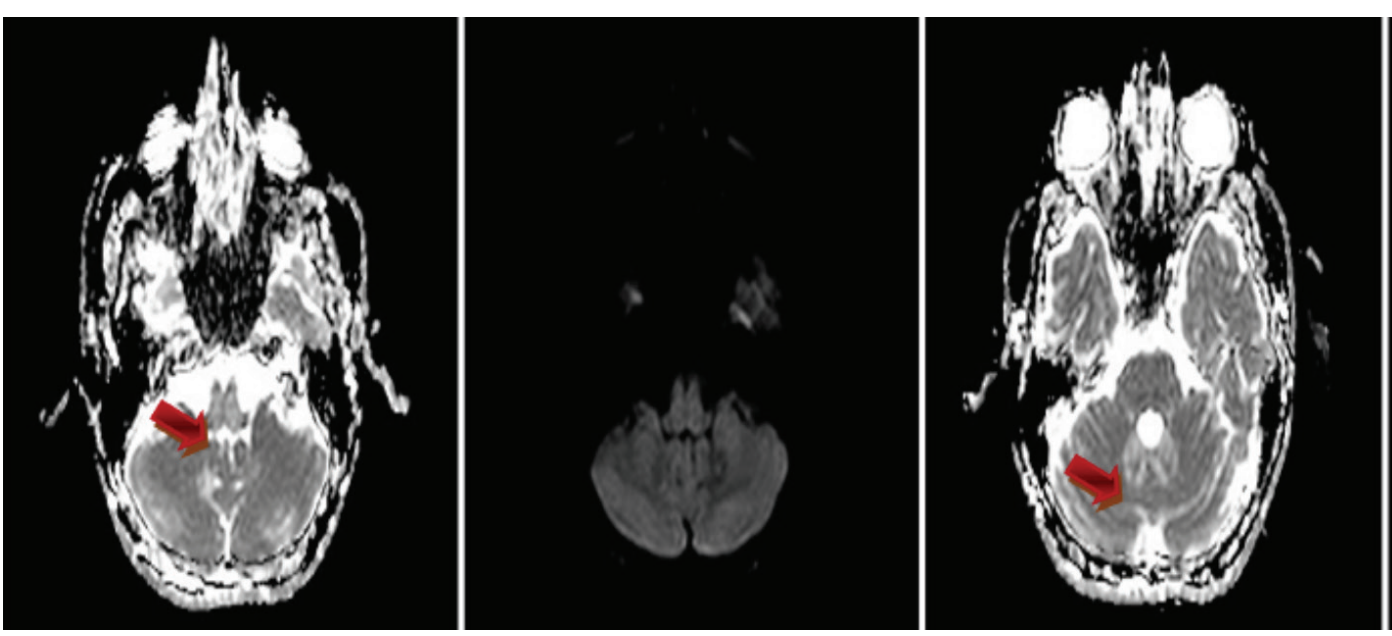

negative. Diffusion-weighted magnetic resonance imaging (MRI) showed a hyperintense signal in the brainstem surrounding the fourth ventricle, without hypointensity on apparent diffusion coefficient map (Figure 1) and cerebral T2-weighted sequence revealed symmetric hyperintense lesions in the pons, bulbus, mesencephalon, and cerebellum, without contrast enhancement (Figure 2).

On the fifth day of hospitalization, the patient was intubated due to respiratory failure. Distal muscle weakness simultaneously developed in all extremities. His ophthalmoparesis progressed to ophthalmoplegia. Due to the patient's poor general condition, electromyographic examination was not performed. He was treated with $400 \mathrm{mg} / \mathrm{kg}$ of intravenous immune globulin (IVIG) for 5 days. Afterward, the patient developed myocarditis and kidney failure. Follow-up MRI showed novel contrast-enhancing lesions on medulla oblongata, cervical medulla, thalamus, and left basal ganglia, as well as a dramatic increase in the size of previous lesions (Figure 3). Treatment was re-planned with

Figure 1. Diffusion-weighted imaging; showed hyperintense signal in the brainstem surrounding the fourth ventricle, without hypointensity on apparent diffusion coefficient map
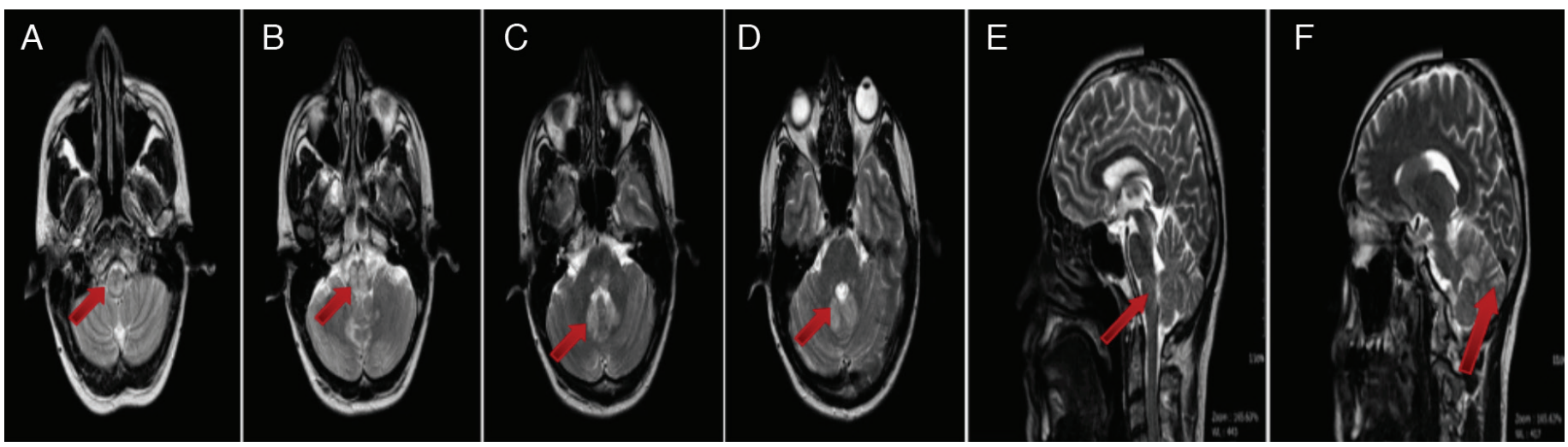

Figure 2. Axial (A-D) and sagittal (E, F) T2-weighted MRI sequences showed symmetric hyperintense lesions in the pons, bulbus, mesencephalon, and cerebellum

MRI: Magnetic resonance imaging 

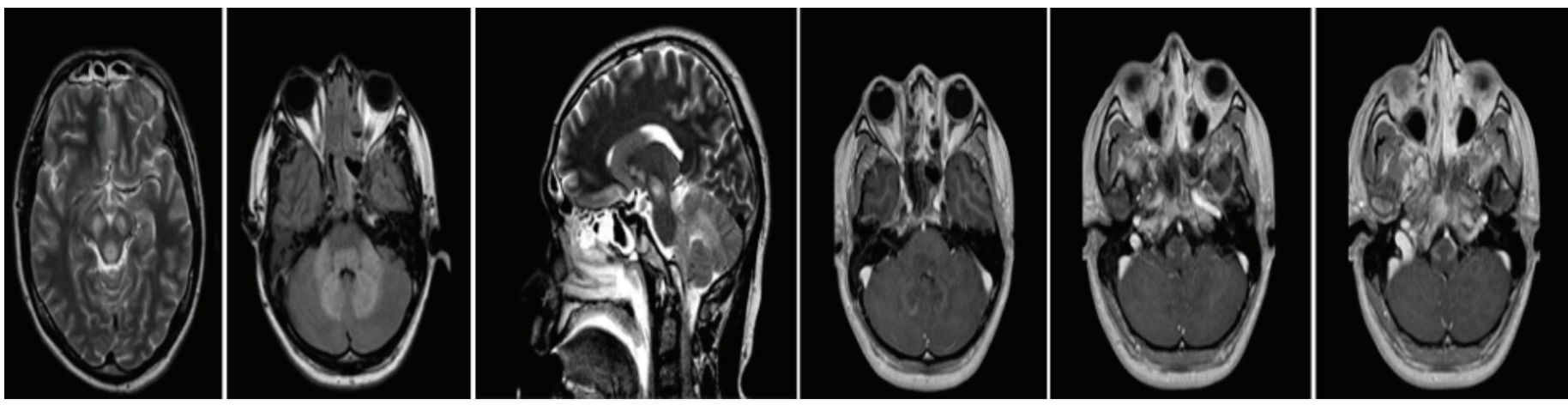

Figure 3. Increase in size of previous lesions with new contrast-enhancing lesions on medulla oblongata, cervical medulla, thalamus, and left basal ganglia

intravenous prednisolone infusion at a dosage of $1000 \mathrm{mg}$ for 5 days. No apparent response was observed from IVIG and pulse prednisolone therapies, thus plasmapheresis was initiated. After the fifth session of plasmapheresis, the patient died due to cardiorespiratory failure.

\section{DISCUSSION}

The mechanisms underlying the neurological damage caused by SARS-CoV-2 are tempting research topics. The presumptive pathophysiological theories are direct viral invasion, postviral triggered immune response (postviral autoimmune process), or hypoxic-metabolic complications due to severe illness itself.

The spike proteins of SARS-CoV-2 use angiotensin-converting enzyme receptor type 2 (ACE-2), which is expressed in lung epithelial cells, heart, arteries, oral and nasal mucosa, and the central nervous system (predominantly in thalamic nuclei, cerebellum, and inferior olivary nuclei) (4). Alveolar type 2 cells, brain endothelial cells, neurons, and glial cells all possess ACE-2 receptors $(5,6)$. Moreover, SARS-CoV-2 uses sialic acid-containing glycoproteins and gangliosides on cell surfaces to bind to cells (7). Endothelial cells of the blood-brain barrier express ACE-2 receptors, which facilitate viral entry into the central nervous system via hematogenous route (3). As a transneuronal route, SARS-CoV-2 infects the nasal epithelium and olfactory receptor neurons in the neuroepithelium, causing neuroinvasion of the olfactory bulb, which explains anosmia, the common and early symptom of SARS-CoV-2 infection (7). Infection with SARSCoV-2 produces increased levels of tumor necrosis factor-alpha, interferon-gamma, as well as interleukin 6, 12, and 15, which is a phenomenon referred to as cytokine storm (8). A combination of this pro-inflammatory process and localized lung injury with severe hypoxia may be the reason for the cerebral vasodilation that leads to cerebral edema and ischemia (3). Acute lung injury and neurotoxicity may be caused by cytokine storms. Cytokinedriven injury and immune-mediated toxicity may disrupt the blood-brain barrier without direct viral invasion. Acute necrotizing encephalopathy may be caused by cytokine toxicity (8). A hypercoagulable state evidenced by increased D-dimer, prolonged prothrombin time, and disseminated intravascular coagulation may complicate COVID-19 by causing acute cerebrovascular disease (4).

Cerebrovascular disease, encephalopathy, and encephalitis, including Bickerstaff encephalitis, impaired level of consciousness, and GBS, are neurologic complications of SARSCoV-2 $(3,9)$. Encephalitis is an acute inflammatory condition of the brain, which is characterized by seizure, focal neurologic deficits, acute onset of fever, vomiting, and altered consciousness. Apart from direct viral invasion of SARS-CoV-2, encephalitis may be implicated in inflammatory and hypoxic-metabolic processes (3). It may be associated with autoimmune and paraneoplastic syndromes.

Miller Fisher, a variant of GBS, is an acute peripheral neuropathy. Miller Fisher syndrome can develop after various viral, bacterial, or fungal pathogens. The syndrome is characterized by a triad of ophthalmoplegia, ataxia, and areflexia and might be associated with anti-GQ1b antibody. Lantos et al. (9) reported a presumptive case of COVID-19 associated with Miller Fisher syndrome. Although the test was negative for anti-GQ1b antibody, the clinical picture was observed to be consistent with Miller Fisher syndrome. A review of 123 patients with Miller Fisher syndrome revealed that $15 \%$ were negative for anti-GQ1b (10). Given these findings, Lantos et al. (9) assumed that when antibody testing is negative, symptoms may be related to viral neurotropism rather than immune-mediated injury. On the other hand, their MRI demonstrated T2-hyperintensity and enhancement of the affected cranial nerve III from the cavernous sinus through 
the orbit. Our patient exhibited multiple cerebral findings due to COVID-19. In our patient, we found not only Miller Fisher syndrome, but rhombencephalitis. The case in Lantos et al.'s (9) report improved after IVIG treatment. However, IVIG, pulse steroid, and plasmapheresis options all failed as a result of the severe clinical and neurological status of our patient. The present case was similar to an example of both central and peripheral involvement of the neurological system due to COVID-19.

Symmetric hyperintense lesions in the pons, bulbus, mesencephalon, and cerebellum were the remarkable MRI findings in our patient. These inflammatory changes in the brainstem and cerebellum prompted us to diagnose rhombencephalitis. On differential diagnosis, we considered Behçet disease, paraneoplastic syndrome, metronidazole intoxication, thiamine abstinence, Wernicke's encephalopathy, and infectious causes, such as listeria monocytogenes, EpsteinBarr virus, herpes, and tuberculosis. Multiple sclerosis, cerebral venous thrombosis, and brain abscess were not initially considered and excluded. Although cerebrospinal fluid PCR was negative for SARS-CoV-2 RNA, nasopharyngeal PCR test was positive. The combination of ophthalmoplegia, areflexia, ataxia, motor deficits/polyneuropathy, and negative cerebrospinal fluid results is considered to be associated with virus-mediated immune response rather than direct viral invasion.

Wong and colleagues reported a 40-year-old man who developed acute brainstem dysfunction 3 days after hospitalization, with symptoms of the novel SARS-CoV-2 infection (COVID-19). MRI showed changes consistent with inflammation of the brainstem and upper cervical cord. The patient had an unsteady gait, diplopia, oscillopsia, and limb ataxia. Unlike our patient, peripheral reflexes were intact. They considered Miller Fisher, a variant of GBS, in their clinical differential diagnosis; however, MRI of the brain and cervical spine suggested an inflammatory rhombencephalitis/myelitis (11).

Zhao et al. (12) reported the first case of COVID-19 that initially occurs with an acute GBS. A 61-year-old woman showed acute weakness and severe fatigue in both legs, which progressed within 1 day. She was diagnosed with GBS and was administered intravenous immunoglobulin treatment. It was reported that the patient's clinical condition gradually improved (12).

GBS has recently been associated with SARS-CoV-2 infection, with five cases reported in Italy and two additional cases from Wuhan, China (3,12-14). All patients experienced a varying prodrome of upper respiratory tract infection 5 to 14 days before the development of symmetrical weakness and 3 patients developed respiratory failure (12-14). All patients had a positive nasopharyngeal PCR and chest imaging of SARS-CoV-2, whereas all cerebrospinal fluid samples had a negative SARS-CoV-2 PCR. While all patients received IVIG, those who developed respiratory failure showed poor results (13). Interestingly, MRI of the brain and spine did not show abnormalities in half of the patients. In published cases, demyelinating-type involvement findings were found in 7 out of 13 patients who were examined by electromyography $(3,10,12-15)$. IVIG was administered to 15 patients, in addition to the antiviral treatment they were receiving. Two cases of Miller Fisher variant and one case of demyelinating-type GBS fully recovered. Other GBS cases improved to varying degrees. However, the exact prognosis is not clear, since the follow-up periods of most patients were short.

This is the first case report indicating the coexistence of Miller Fisher syndrome and rhombencephalitis due to COVID-19. As demonstrated in our unusual case, care should be taken against the development of multisystem inflammatory response and neurological symptoms in COVID-19 over time.

\section{CONCLUSION}

COVID-19 may affect the central and peripheral nervous systems. Further studies are needed to elucidate whether the neurological manifestations might occur as result of an aberrant immune response to COVID-19.

\section{Ethics}

Informed Consent: Informed written consent was obtained from the patient.

Peer-review: Externally peer-reviewed.

\section{Authorship Contributions}

Concept: O.A., B.Y., Design: O.A., B.Y., Data Collection or Processing: O.A., B.Y., Analysis or Interpretation: O.A., B.Y., Literature Search: O.A., B.Y., Writing: O.A., B.Y., S.K., F.S., Ș.V., K.H., S.Ü.

Conflict of Interest: No conflict of interest was declared by the authors.

Financial Disclosure: The authors declared that this study received no financial support.

\section{REFERENCES}

1. Huang C, Wang Y, Li X, Ren L, Zhao J, Hu Y, et al. Clinical features of patients infected with 2019 novel coronavirus in Wuhan, China. Lancet 2020;395:497-506.

2. Wang D, Hu B, Hu C, Zhu F, Liu X, Zhang J, et al. Clinical characteristics of 138 hospitalized patients with 2019 novel coronavirus-infected pneumonia in Wuhan, China. JAMA 2020;323:1061-9. 
3. Bridwell R, Long B, Gottlieb M. Neurologic complications of COVID-19. Am J Emerg Med 2020;38:1549.e3-1549.e7.

4. Berger JR. COVID-19 and the nervous system. J Neurovirol 2020;26:1438.

5. Hamming I, Timens W, Bulthuis ML, Lely AT, Navis G, van Goor H. Tissue distribution of ACE2 protein, the functional receptor for SARS coronavirus. A first step in understanding SARS pathogenesis. J Pathol 2004;203:631-7.

6. Doobay MF, Talman LS, Obr TD, Tian X, Davisson RL, Lazartigues E. Differential expression of neuronal ACE2 in transgenic mice with overexpression of the brain renin-angiotensin system. Am J Physiol Regul Integr Comp Physiol 2007;292:R373-81.

7. Dalakas, MC. Guillain-Barré syndrome: The first documented COVID19-triggered autoimmune neurologic disease. Neurol Neuroimmunol Neuroinflamm 2020;7:e781.

8. Aghagoli G, Marin BG, Katchur NJ, Chaves-Sell F, Asaad WF, Murphy SA. Neurological involvement in COVID-19 and potential mechanisms: A review. Neurocritical Care 2020;13:1-10.

9. Lantos JE, Strauss SB, Lin E. COVID-19-Associated Miller Fisher Syndrome: MRI Findings. AJNR Am J Neuroradiol 2020;41:1184-6.
10. Nishimoto Y, Odaka M, Hirata K, Yuki N. Usefulness of anti-GQ1b IgG antibody testing in Fisher syndrome compared with cerebrospinal fluid examination. J Neuroimmunol 2004;148:200-5.

11. Wong PF, Craik S, Newman P, Makan A, Srinivasan K, Crawford E, et al. Lessons of the month 1: A case of rhombencephalitis as a rare complication of acute COVID-19 infection. Clin Med (Lond) 2020;20:2934.

12. Zhao H, Shen D, Zhou H, Liu J, Chen S. Guillain-Barré syndrome associated with SARS-CoV-2 infection: causality or coincidence? Lancet Neurol 2020;19:383-4.

13. Toscano G, Palmerini F, Ravaglia S, Ruiz L, İnvernizzi P, Cuzzoni MG, et al. Guillain-Barré Syndrome Associated with SARS-CoV-2. N Engl J Med 2020;382:2574-6

14. Sedaghat Z, Karimi N. Guillain Barre syndrome associated with COVID-19 infection: A case report. J Clin Neurosci 2020;76:233-5.

15. Gutiérrez-Ortiz C, Méndez-Guerrero A, Rodrigo-Rey S, Pedro-Murillo ES, Bermejo-Guerrero L, Gordo-Mañas R, et al. Miller Fisher syndrome and polyneuritis cranialis in COVID-19. Neurology 2020;95:601-5. 